\title{
A NOTE ON THE PROBLEM OF PRESCRIBING GAUSSIAN CURVATURE ON SURFACES
}

\author{
WEI-YUE DING AND JIA-QUAN LIU
}

\begin{abstract}
The problem of existence of conformal metrics with Gaussian curvature equal to a given function $K$ on a compact Riemannian 2-manifold $M$ of negative Euler characteristic is studied. Let $K_{0}$ be any nonconstant function on $M$ with $\max K_{0}=0$, and let $K_{\lambda}=K_{0}+\lambda$. It is proved that there exists a $\lambda^{*}>0$ such that the problem has a solution for $K=K_{\lambda}$ iff $\lambda \in\left(-\infty, \lambda^{*}\right]$. Moreover, if $\lambda \in\left(0, \lambda^{*}\right)$, then the problem has at least 2 solutions.
\end{abstract}

Let $M$ be a closed 2-dimensional smooth manifold and $g$ be a Riemannian metric on $M$. Let $k$ denote the Gaussian curvature of $g$. If $g^{\prime}=e^{2 u} g$ is another Riemannian metric conformal to $g$, and has Gaussian curvature $k^{\prime}$, then it is well known that

$$
k^{\prime}=e^{-2 u}(k-\Delta u),
$$

where $\Delta$ is the Laplacian of $g$. Given a function $K \in C^{\infty}(M)$, the problem of prescribing Gaussian curvature asks whether one can find $u \in C^{\infty}(M)$ such that the metric $g^{\prime}=e^{2 u} g$ has the given $K$ as its Gaussian curvature. Obviously, this is equivalent to the problem of solvability of the following elliptic equation

$$
\Delta u-k+K e^{2 u}=0, \quad \text { on } M .
$$

If $u$ is a solution of (1), then we have by integrating (1)

$$
\int_{M} K e^{2 u} d v=\int_{M} k d v
$$

where $d v$ is the area element with respect to the metric $g$. It follows from the Gauss-Bonnet formula that

$$
\int_{M} k e^{2 u} d v=2 \pi \chi(M),
$$

where $\chi(M)$ is the Euler characteristic of $M$. Note that (2) poses restrictions on the given function $K$ for the solvability of (1), according to the sign of $\chi(M)$.

If $\chi(M)=0$, the problem of the solvability of (1) has been completely resolved. (See [K-W].) If $\chi(M)>0$, then $M$ is either $R P^{2}$ (the real projective

Received by the editors December 8,1993; originally communicated to the Proceedings of the $A M S$ by Peter Li.

1991 Mathematics Subject Classification. Primary 58G03.

Research of both authors was supported in part by the National Natural Science Foundation of China.

(C)1995 American Mathematical Society 
plane) or $S^{2}$ (the 2-sphere). While the case where $M=R P^{2}$ has been well understood (see [M], [A]), the case where $M=S^{2}$ is much more complicated. Many authors have studied the problem on $S^{2}$ with its standard metric, known as Nirenberg problem (see e.g. [C-D], [C-Y1, 2], [C-L]).

In this note we consider only the case where $\chi(M)<0$; in other words,

$$
\int_{M} k d v<0 .
$$

This case has been studied by Kazdan and Warner in [K-W] using the method of super- and sub-solutions for second order elliptic equations. The following are some facts derived by them.

Fact (i). One can always find an arbitrarily negative subsolution $\varphi$ for equation (1). Indeed, such a subsolution can be of the form $\varphi_{c}=f-c$, where $f$ is a solution to the equation $\Delta f=k-\bar{k}$ with $\bar{k}$ being the mean value of $k$, and $c$ is any sufficiently large number. Therefore, to solve (1) one needs only to find a supersolution $\psi$ for (1).

Fact (ii). Let $K_{1} \geq K_{2}$ are two smooth functions on $M$. Suppose that (1) has a solution $u_{1}$ for $K=K_{1}$. Then, since $u_{1}$ is a supersolution for (1) with $K=K_{2}$ as can be easily checked, we see that (1) is solvable for $K=K_{2}$ by Fact (i).

Fact (iii). It is easy to see from (2) that a necessary condition for (1) to be solvable is that the function $K$ is negative somewhere on $M$. On the other hand, if $K \leq 0$, then one can find a supersolution for (1). It follows from Fact (i) that (1) has a solution provided $K \leq 0$. Moreover, in such a case, one can show that the solutions of (1) are unique.

In view of Fact (iii), we are only interested in the case where the function $K$ changes sign. From now on, we assume that $K_{0} \in C^{\infty}(M)$ is a nonconstant function which satisfies

$$
\operatorname{Max}_{x \in M} K_{0}(x)=0
$$

and let $K_{\lambda}=K_{0}+\lambda$, where $\lambda$ is a real number. Consider the family of equations

$$
\Delta u-k+K_{\lambda} e^{2 u}=0 \text {. }
$$

By Fact (iii), (1) $\lambda$ has a unique solution $u_{\lambda}$ for $\lambda \leq 0$. On the other hand, for the solution $u_{0}$ of $(1)_{0}$, the variational equation

$$
\Delta v+2 K_{0} e^{2 u_{0}} v=0
$$

has only a trivial solution $v \equiv 0$, since $K_{0} \leq 0$ and $K_{0} \not \equiv 0$. It follows from the implicit function theorem that $(1)_{\lambda}$ has a solution for sufficiently small $\lambda>0$. So we have

Lemma 1. There exists $a \lambda^{*}>0$ such that (1) $\lambda$ is solvable for all $\lambda<\lambda^{*}$, and it has no solutions for $\lambda>\lambda^{*}$.

Proof. Let $\lambda^{*}$ be the supremum of all $\lambda$ for which $(1)_{\lambda}$ has a solution. We have known that $\lambda^{*}<0$, and $\lambda^{*}<-\inf _{M} K_{0}$ by (iii). It follows from Fact (ii) that $\lambda^{*}$ has the claimed property.

Our main result is as follows. 
Theorem. Let $K_{0} \in C^{\infty}(M)$ be any nonconstant function satisfying (3), and let $K_{\lambda}=K_{0}+\lambda$. Then there exists a $\lambda^{*}>0$ such that (a) (1) has a unique solution for $\lambda \leq 0$; (b) (1) has at least two solutions if $0<\lambda<\lambda^{*}$; and (c) (1) $\lambda^{*}$ has at least one solution.

Remark. If we set

$$
S=\left\{K \in C^{\infty}(M):(1) \text { is solvable }\right\},
$$

then the Theorem implies that the set $S \cup\{0\}$ is closed in $C^{0}$ topology. Indeed, let $\left\{K_{i}\right\} \subset S$ be a sequence such that $K_{i} \rightarrow K \in C^{\infty}(M) \backslash\{0\}$. Then for any $\varepsilon>0$ we can find $K_{i}$ such that $K-\varepsilon \leq K_{i}$, and this shows that $K-\varepsilon \in S$ for any $\varepsilon>0$. It follows from (c) of the Theorem that $K \in S$.

Now we turn to the proof of the Theorem. It is clear that conclusion (a) follows from Fact (iii). Hence we need only prove (b) and (c).

Proof of $(b)$ of the Theorem. Note that $(1)_{\lambda}$ is the Euler-Lagrange equation of the functional

$$
I_{\lambda}(u)=\int_{M}\left(|\nabla u|^{2}+2 k u-K_{\lambda} e^{2 u}\right) d v .
$$

We are to apply variational methods (see [C]) to obtain multiple critical points for $I_{\lambda}$, which correspond to solutions of $(1)_{\lambda}$, for $\lambda \in\left(0, \lambda^{*}\right)$. Fixing any $\lambda \in\left(0, \lambda^{*}\right)$, we choose a $\lambda_{1} \in\left(\lambda, \lambda^{*}\right)$. Let $\psi$ be a solution of $(1)_{\lambda_{1}}$. Then $\psi$ is a super-solution for the equation $(1)_{\lambda}$. By Fact (i), we can find a sub-solution $\varphi$ for $(1)_{\lambda}$ such that $\varphi<\psi$ on $M$. Let $[\varphi, \psi]$ be the order interval defined by

$$
[\varphi, \psi]=\left\{v \in C^{1}(M): \varphi \leq v \leq \psi \text { on } M\right\} .
$$

The ordinary super- and sub-solution method asserts that $(1)_{\lambda}$ has a solution $u_{\lambda} \in[\varphi, \psi]$. Further variational considerations as in [C] permits one to assume that $u_{\lambda}$ is $I_{\lambda}$-minimizing in the interval $[\varphi, \psi]$, i.e.,

$$
I_{\lambda}\left(u_{\lambda}\right)=\inf \left\{I_{\lambda}(v): v \in[\varphi, \psi]\right\} .
$$

Next, we note that there exist functions $w \in C^{1}(M)$ such that $I_{\lambda}(w)<I_{\lambda}\left(u_{\lambda}\right)$. Indeed, since $\lambda>0$, the set $M_{\varepsilon}=\left\{x \in M: K_{\lambda}(x)>\varepsilon\right\}$ for small $\varepsilon>0$ is nonempty and open. Let $f \in X$ be any function which is positive in $M_{\varepsilon}$ and vanishes on $M \backslash M_{\varepsilon}$. Then

$$
\begin{aligned}
I_{\lambda}(t f) & =t^{2} \int_{M}|\nabla f|^{2} d v+t \int_{M} k f d v-\int_{M_{\varepsilon}} K_{\lambda} e^{2 t f} d v-\int_{M \backslash M_{\varepsilon}} K_{\lambda} d v \\
& \leq A t^{2}+B t+C-\varepsilon \int_{M_{\varepsilon}} e^{2 t f} d v \\
& \leq A t^{2}+B t+C-a \varepsilon e^{2 t a^{-1} \int_{M_{\varepsilon}} f d v} \rightarrow-\infty, \quad \text { as } t \rightarrow \infty,
\end{aligned}
$$

where $a$ is the area of $M_{\varepsilon}$. Thus, we may take $w=t f$ with $t$ big enough. Now, if the functional satisfies the Palais-Smale condition, a result of K. C. Chang [C] asserts the existence of a mountain-pass critical point $v_{\lambda}$ other than $u_{\lambda}$. The fact that $I_{\lambda}$ does satisfy the Palais-Smale condition is proved in the next lemma. This completes the proof of $(b)$. 
Lemma 2. Assume that the set $M_{-}=\left\{x \in M: K_{\lambda}(x)<0\right\}$ is nonempty. Then the functional $I_{\lambda}$ satisfies the Palais-Smale condition in the function space $X=$ $W^{1,2}(M)$. That is to say, if $\left\{u_{k}\right\}$ is any sequence in $X$ such that $I_{\lambda}\left(u_{k}\right) \rightarrow c$ for some $c \in R$ and $I_{\lambda}^{\prime}\left(u_{k}\right) \rightarrow 0$ in $X^{*}$ (the dual space of $X$ ), then a subsequence of $\left\{u_{k}\right\}$ converges in $X$.

Proof. Let $\left\{u_{k}\right\}$ be the sequence in the lemma. Then we have

$$
I_{\lambda}\left(u_{k}\right)=\int_{M}\left(\left|\nabla u_{k}\right|^{2}+2 k u_{k}-K_{\lambda} e^{2 u_{k}}\right) d v \rightarrow c,
$$

and

$$
I_{\lambda}^{\prime}\left(u_{k}\right)(\varphi)=\int_{M}\left(\nabla u_{k} \cdot \nabla \varphi+k \varphi-K_{\lambda} e^{2 u_{k}} \varphi\right) d v=o(\|\varphi\|), \quad \forall \varphi \in X,
$$

where $\|\cdot\|$ is the norm of $X$. Let $u_{k}^{+}=\max \left\{u_{k}, 0\right\}$. We claim that $\left\{u_{k}^{+}\right\}$ is locally $W^{1,2}$-bounded in the open set $M_{-}$. More precisely, we will prove that for any domain $\Omega \subset M_{-}$with $\operatorname{dist}\left(\Omega, \partial M_{-}\right)=d(\Omega)>0$, we have $\left\|u_{k}^{+}\right\|_{W^{1,2}(\Omega)} \leq C$, where the constant $C$ depends only on $d(\Omega)$. To see that our claim holds it suffices to show that for any $p \in M_{-}$with $\operatorname{dist}\left(p, \partial M_{-}\right)=d$, we have

$$
\int_{B_{d / 4}}\left(\left|\nabla u_{k}^{+}\right|^{2}+\left(u_{k}^{+}\right)^{2}\right) d v \leq C,
$$

where $B_{r}$ denote the geodesic ball centered at $p$ with radius $r>0$, and the constant $C>0$ depends only on the distance $d$. To prove (7), let $\eta$ be a smooth cut-off function supported in $B_{d / 2}=B_{d / 2}(p)$, such that $\eta(x)=1$ for $x \in B_{d / 4}, \eta(x)=0$ for $x \in M \backslash B_{d / 2}$ and $0 \leq \eta \leq 1,|\nabla \eta| \leq A d^{-1}$ on $M$. Substituting $\varphi=\eta^{2} u_{k}^{+}$in (6) we get

$$
\int_{B_{d / 2}}\left(\nabla u_{k}^{+} \cdot \nabla\left(\eta^{2} u_{k}^{+}\right)+k \eta^{2} u_{k}^{+}-K_{\lambda} e^{2 u_{k}^{+}} \eta^{2} u_{k}^{+}\right) d v \leq C\left\|\eta^{2} u_{k}^{+}\right\| \leq C\left\|\eta u_{k}^{+}\right\| .
$$

Here and in the sequel we use $C$ to denote various constants depending only on $d$. Using

$$
\begin{gathered}
\nabla u_{k}^{+} \cdot \nabla\left(\eta^{2} u_{k}^{+}\right)=\left|\nabla\left(\eta u_{k}^{+}\right)\right|^{2}+|\nabla \eta|^{2}\left(u^{+}\right)^{2}, \\
K_{\lambda} \leq-\varepsilon \text { in } B_{d / 2} \text { for some } \varepsilon>0, \text { and } e^{2 t} \geq t^{3} \text { for } t \in R,
\end{gathered}
$$

we derive from (8) that

$$
\int_{B_{d / 2}}\left(\left|\nabla\left(\eta u_{k}^{+}\right)\right|^{2}+\varepsilon \eta^{2}\left(u_{k}^{+}\right)^{4}\right) d v \leq-\int_{B_{d / 2}} k \eta^{2} u_{k}^{+} d v+C\left\|\eta u_{k}^{+}\right\| .
$$

Since $\left(u_{k}^{+}\right)^{4}>\left(u_{k}^{+}\right)^{2}-1$, it is easy to see from the above inequality that

$$
\varepsilon\left\|\eta u_{k}^{+}\right\|^{2} \leq C\left\|\eta u_{k}^{+}\right\|+C .
$$

From this it follows that $\left\|\eta u_{k}^{+}\right\| \leq C$, and consequently (7) holds since $\eta \equiv 1$ in $B_{d / 4}$. Next, letting $\varphi \equiv 1$ in (6) we have

$$
\int_{M} K_{\lambda} e^{2 u_{k}} d v-\int_{M} k d v \rightarrow 0, \quad \text { as } k \rightarrow \infty .
$$


Combining with (5), this gives that

$$
\int_{M}\left(|\nabla u|^{2}+2 k u_{k}\right) d v=I_{\lambda}\left(u_{k}\right)-\int_{M} k_{\lambda} e^{2 u_{k}} d v \rightarrow c-2 \pi \chi(M),
$$

as $k \rightarrow \infty$.

Now we claim that $\left\{u_{k}\right\}$ is bounded in $L^{2}(M)$. If the claim is true, then (10) implies that $\left\{u_{k}\right\}$ is also bounded in $X=W^{1,2}(M)$. By passing to a subsequence if necessary we may assume that $u_{k}$ converge weakly in $X$ to some $u_{0}$. Then it is standard to show that $u_{k}$ actually converge strongly in $X$ using (6) and the fact that $e^{2 u_{k}} \rightarrow e^{2 u_{0}}$ in $L^{p}(M)$ for any $p \geq 1$. (Note that $\operatorname{dim} M=2$.) This will finish our proof of Lemma 2 .

To prove our claim we assume that on the contrary, $\left\|u_{k}\right\|_{L^{2}(M)} \rightarrow \infty$ and consider $v_{k}=u_{k} /\left\|u_{k}\right\|_{L^{2}}$, which satisfy $\left\|v_{k}\right\|_{L^{2}}=1$ for all $k$. We see from (10) that

$$
\int_{M}\left|\nabla v_{k}\right|^{2} d v=-2 \int_{M} k \frac{v_{k}}{\left\|u_{k}\right\|_{L^{2}}} d v+o(1) \rightarrow 0 .
$$

It follows that $v_{k}$ converges in $X$ to some constant function $v \equiv \beta$. Since $\|v\|_{L^{2}}=1$ we have $\beta \neq 0$. Note that (10) also implies that

$$
\int_{M} k v_{k} d v \leq C\left\|u_{k}\right\|_{L^{2}}^{-1}
$$

Taking the limit we get that

$$
\int_{M} \beta k d v=2 \pi \chi(M) \beta \leq 0 .
$$

Since $\beta$ is nonzero and $\chi(M)<0$, we must have $\beta>0$. Now, consider $v_{k}^{+}=u_{k}^{+} /\left\|u_{k}\right\|_{L^{2}}$. The above discussion shows that $v_{k}^{+}$converge to $\beta>0$ almost everywhere in $M$. However, as we have proved, $u_{k}^{+}$is locally $W^{1,2}$ bounded in $M_{-}$, which implies that $v_{k}^{+}$converge to 0 almost everywhere in $M_{-}$, a contradiction! This completes our proof of Lemma 2.

We now turn to

Proof of $(\mathrm{c})$ of the Theorem. We are to prove that $(1)_{\lambda}$. has a solution. This will be proven by showing that certain solutions of (1) $)_{\lambda}$ converge in $X$ as $\lambda \rightarrow \lambda^{*}$.

We have seen in the proof of (b) that for $\lambda<\lambda^{*},(1)_{\lambda}$ has a solution $u_{\lambda}$ which is $I_{\lambda}$-minimizing in an order interval $[\varphi, \psi]$ in $C^{1}(M)$ (see (4)). By the maximum principle, we must have $\varphi<u_{\lambda}<\psi$. This implies that $u_{\lambda}$ is a local minima for $I_{\lambda}$ in $C^{1}(M)$. It follows that the second variation of $I_{\lambda}$ at $u_{\lambda}$ is nonnegative, i.e.,

$$
\int_{M}\left(|\nabla \varphi|^{2}-2 K_{\lambda} e^{2 u_{\lambda}} \varphi^{2}\right) d v \geq 0,
$$

where $\varphi \in C^{1}(M)$. We also note that there is a $C>0$ such that for $\lambda \in\left(0, \lambda^{*}\right)$

$$
u_{\lambda} \geq-C, \quad \text { on } M \text {. }
$$

Actually, let $\varphi_{c}=f-c$ be the family of functions in Fact (i). Then for $c \geq$ some $c_{0}, \varphi_{c}$ is a continuous family of subsolutions for $(1)_{0}$, hence it is also a continuous family of subsolutions for $(1)_{\lambda}$, where $\lambda \in\left(0, \lambda^{*}\right)$. We claim that 
$u_{\lambda} \geq \varphi_{c_{0}}$, and consequently (12) holds. For otherwise, by varying $c \in\left[c_{0}, \infty\right)$, we find that for some $c$ we have

$$
u_{\lambda} \geq \varphi_{c} \text { on } M, \text { and } u_{\lambda}\left(x_{0}\right)=\varphi_{c}\left(x_{0}\right) \text { for some } x_{0} \in M \text {. }
$$

This, by the maximum principle, can occur only if $u_{\lambda} \equiv \varphi_{c}$, which is impossible. So we see that (12) holds.

The crucial point of this proof is to show that $u_{\lambda}$ is uniformly bounded in $X$ as $\lambda \rightarrow \lambda^{*}$. If this is true, then by elliptic $L^{p}$-estimate for the solutions of $(1)_{\lambda}$ we see that $u_{\lambda}$ is uniformly bounded in $W^{2, p}(M)$ for any $p>1$. The Sobolev imbedding theorem together with Schauder estimates then imply that $u_{\lambda}$ is uniformly $C^{2, \alpha}$-bounded. It follows that some subsequence of $u_{\lambda}$ converges in $C^{2}$ to a solution of $\lambda^{*}$. This will complete our proof. We now proceed to prove the $W^{1,2}$-boundedness of $u_{\lambda}$. To this end we need to use the conformal invariance of equation (1). Note that $u_{\lambda}$ being a solution of $(1)_{\lambda}$ is equivalent to the Gaussian curvature of $g_{\lambda}=e^{2 u_{i}} g$ being $K_{\lambda}$. If $g^{\prime}=e^{2 v} g$ is any metric conformal to $g$, then we have $g_{\lambda}=e^{2\left(u_{\lambda}-v\right)} g^{\prime}$. This means that the function $w_{\lambda}=u_{\lambda}-v$ solves

$$
\Delta_{g^{\prime}} w-k_{g^{\prime}}+K_{\lambda} e^{2 w}=0,
$$

where $\Delta_{g^{\prime}}$ and $k_{g^{\prime}}$ are respectively the Laplacian and Gaussian curvature of $g^{\prime}$.

Claim. The set $M_{-}^{*}=\left\{x \in M: K_{\lambda^{*}}(x)<0\right\}$ is nonempty. We choose $g^{\prime}$ in (13) to be the uniqueness metric $g_{0}=e^{2 v_{0}} g$ which has constant curvature $k_{0} \equiv-1$, where $v_{0}$ is the unique solution of $\Delta v-k-e^{2 v}=0$. Then $w_{\lambda}=u_{\lambda}-v_{0}$ is a solution of

$$
\Delta_{0} w+1+K_{\lambda} e^{2 w}=0 .
$$

Here and in the sequel, by the subscript 0 we mean that the corresponding geometric objects are for the metric $g_{0}$. Multiplying (14) by $e^{-2 w_{\lambda}}$ and integrating over $M$ we get

$$
\int_{M} K_{\lambda} d v_{0}=-\int_{M}\left(2\left|\nabla w_{\lambda}\right|_{0}^{2}+1\right) e^{-2 w_{\lambda}} d v_{0} .
$$

Letting $\lambda \rightarrow \lambda^{*}$ we see that $\int K_{\lambda^{*}} \leq 0$. If the Claim is false then we must have $K_{\lambda^{*}} \geq 0$, and consequently $K_{\lambda^{*}} \equiv 0$. This contradicts our assumption that $K_{\lambda}$ are nonconstant for all $\lambda$, showing that the Claim is true.

Now, let $h$ be a smooth function which vanishes outside an open set $D$ such that $\bar{D} \subset M_{-}^{*}$ and $h<0$ in $D$. As in the proof of (b) of the Theorem, one may derive that $u_{\lambda}^{+}$is uniformly bounded in $W^{1,2}(D)$ for $\lambda \in\left(0, \lambda^{*}\right)$, and hence by a variant of the Moser-Trudinger inequality (see [C-Y2, p. 271]) we have

$$
\int_{D} e^{2 u_{i}} \leq C
$$

Next, let $g_{1}=e^{2 v_{1}} g$ be the metric with Gaussian curvature $h$, where $v_{1}$ is the unique solution of the equation $\Delta v-k+h e^{2 v}=0$. Then the function $w_{\lambda}=u_{\lambda}-v_{1}$ satisfies the equation

$$
\Delta_{1} w_{\lambda}-h+K_{\lambda} e^{2 w_{i}}=0 .
$$


Since $\Delta_{1}=e^{-2 v_{1}} \Delta$, we have

$$
\Delta w_{\lambda}-h e^{2 v_{1}}+K_{\lambda} e^{2\left(w_{\lambda}+v_{1}\right)}=0 .
$$

Multiplying (16) by $e^{2 w_{\lambda}}$ and integrating over $M$ gives

$$
2 \int_{M}\left|\nabla e^{w_{\lambda}}\right|^{2} d v+\int_{M} h e^{2 v_{1}} e^{2 w_{\lambda}} d v-\int_{M} K_{\lambda} e^{2 v_{1}} e^{4 w_{\lambda}} d v=0 .
$$

On the other hand, letting $\varphi=e^{w_{\lambda}}$ in (11) we have

$$
\int_{M}\left|\nabla e^{w_{\lambda}}\right|^{2} d v-2 \int_{M} K_{\lambda} e^{2 v_{1}} e^{4 w_{\lambda}} \geq 0 .
$$

Together with (17) this gives

$$
\int_{M}\left|\nabla e^{w_{\lambda}}\right|^{2} d v \leq-\frac{2}{3} \int_{M} h e^{2\left(w_{\lambda}+v_{1}\right)} d v=-\frac{2}{3} \int_{D} h e^{2 u_{\lambda}} d v .
$$

Thus, by (15), $\left|\nabla e^{w_{\lambda}}\right|$ is uniformly bounded in $L^{2}(M)$. We claim that $\left\|e^{w_{\lambda}}\right\|_{L^{2}(M)}$ is uniformly bounded too, consequently $e^{w_{\lambda}}$ is uniformly bounded in $X$. In fact, if this is not true, we may assume that $\left\|e^{w_{\lambda}}\right\|_{L^{2}} \rightarrow \infty$ as $\lambda \rightarrow \lambda^{*}$. Set

$$
v_{\lambda}=e^{w_{\lambda}} /\left\|e^{w_{\lambda}}\right\|_{L^{2}}
$$

Then we have

$$
\left\|v_{\lambda}\right\|_{L^{2}}=1, \quad \text { and }\left\|\nabla v_{\lambda}\right\|_{L^{2}} \rightarrow 0 .
$$

It follows that $v_{\lambda}$ converges in $X$ to a constant function $v$ with $\|v\|_{L^{2}}=1$. However, (15) implies that $\left\|v_{\lambda}\right\|_{L^{2}(D)} \rightarrow 0$ as $\lambda \rightarrow \lambda^{*}$, and hence $v \equiv 0$ in $D$. But, $v$ is constant on $M$, so $v \equiv 0$ on $M$, contradicting $\|v\|_{L^{2}}=1$. This proves that $e^{w_{\lambda}}$ and also $e^{u_{\lambda}}$ are uniformly bounded in $L^{2}$. Actually, $e^{u_{\lambda}}$ is uniformly $L^{p}$-bounded for any $p>1$ since it is bounded in $X$.

Now we observe that since $u_{\lambda}$ is bounded below by (12), the $L^{p}$-boundedness of $e^{u_{\lambda}}$ implies the $L^{p}$-boundedness of $u_{\lambda}$. Therefore, the elliptic $L^{p}$ and Schauder estimates for the solutions of $(1)_{\lambda}$ lead to a uniform $C^{2, \alpha}$-bound for $u_{\lambda}$. It follows that some subsequence of $u_{\lambda}$ converges in $C^{2}$ to a solution of $(1)_{\lambda}$. This completes the proof of (c) of the Theorem.

\section{REFERENCES}

[A] T. Aubin, Nonlinear analysis on manifolds Monge-Ampere equations, Springer-Verlag, Berlin, 1982.

[C-Y1] S. Y. A. Chang and P. Yang, Prescribing Gaussian curvatures on $S^{2}$, Acta Math. 159 (1987), 214-259.

[C-Y2] _ Conformal deformation of metrics on $S^{2}$, J. Differential Geometry 27 (1988), 259-296.

[C] K. C. Chang, Infinite dimensional Morse theory and multiple solution problems, Birkhäuser, 1992.

[C-L] K. C. Chang and J. Q. Liu, On Nirenberg's problem, Internat. J. Math. 4 (1993), 35-58.

[C-D] W. X. Chen and W. Y. Ding, Scalar curvatures on $S^{2}$, Trans. Amer. Math. Soc. 303 (1987), 365-382. 
[K-W] J. L. Kazdan and F. W. Warner, Curvature functions for compact 2-manifolds, Ann. of Math. 99 (1974), 14-47.

[M] J. Moser, On a nonlinear problem in differential geometry (M. Peixoto, ed.), Dynamical Systems, Academic Press, New York, 1973.

Institute of Mathematics, ACademia Sinica, Beijing, China 100080

Department of Mathematics, Graduate School, Academia Sinica \& USTC, Beijing, CHINA 100039

Current address: Department of Mathematics, Beijing University, Beijing, China 100871 